\title{
Long range diffusion noise in platinum microwires with metallic adhesion layers
}

\author{
Z. Moktadir ${ }^{a)}$ \\ School of Electronics and Computer Science, Southampton University, 5017 1BJ, United Kingdom \\ J. W. van Honschoten and M. Elwenspoek \\ Transducers Science and Technology MESA + Research Institute, University of Twente, 7500 AE Enschede, \\ The Netherlands
}

(Received 26 March 2007; accepted 14 May 2007; published online 6 June 2007)

\begin{abstract}
Voltage fluctuations of platinum wires hosted by silicon nitride beams were investigated. The authors considered four variants of the wires: three with an adhesion layer and one without an adhesion layer. They found that the presence of an adhesion layer changes the nature of the power spectrum which is $1 / f$ for wires with no adhesion layers and $1 / f^{3 / 2}$ for wires with an adhesion layer. They attribute the value of the exponent $\alpha=3 / 2$ found in wires with adhesion layers to the long range diffusion of oxygen atoms along the interface between the adhesion layer and the platinum layer. (C) 2007 American Institute of Physics. [DOI: 10.1063/1.2746961]
\end{abstract}

Low frequency electrical noise in metallic films has been extensively investigated. ${ }^{1-3}$ In a variety of metallic films, the resulting noise spectra are frequency dependent and are known as $1 / f^{\alpha}$ spectrum with the exponent $\alpha \simeq 1$. An important body of research into the origin $1 / f$ noise has emerged but a unifying picture is still lacking. In the Dutta-Horn picture, ${ }^{1} 1 / f$ noise arises from equilibrium defect fluctuations associated with the defects' motion which is thermally activated and nondiffusive. These fluctuations have a characteristic time scale $t_{c}$ which is itself sampled from a distribution function. Other spectra of the form $1 / f^{\alpha}$ were $\alpha \neq 1$ were also found. In particular, the value of $\alpha=3 / 2$ was attributed to transport noise such as long range diffusion in metallic films ${ }^{4-6}$ with hydrogen impurities. This form of the spectrum was also found in silver films subject to electromigration damage, ${ }^{7}$ where it was argued that $1 / f^{3 / 2}$ spectrum is attributed to the long-range diffusion of atoms through pathways opened during electromigration damage.

The present work is concerned with the investigation of the noise spectrum of a velocity sensor. ${ }^{8}$ The sensor consists of two freely suspended silicon nitride beams on the top of which a double layer of platinum and an adhesive metallic layer (adhesion layer) are deposited by sputtering. We show that the presence of the adhesion layer changes the nature of the power spectrum of the voltage fluctuations of the wires. Without the adhesion layer, the low frequency noise follows a power law with the usual power spectrum $1 / f^{\alpha}$, with $\alpha \simeq 1$. The presence of the adhesion layer, changes this power spectrum to $1 / f^{\alpha}$ with $\alpha \simeq 3 / 2$. We attribute this effect to the long range diffusion of oxygen atoms along the interface between the adhesion layer and the platinum.

To fabricate the velocity sensor, we consider four variants: three variants were made by depositing a $10 \mathrm{~nm}$ adhesion layer of chromium $(\mathrm{Cr})$, titanium (Ti), and tantalum(Ta), respectively, followed by a deposition of $150 \mathrm{~nm}$ of platinum. These variants will be refererd to as $\mathrm{Pt} / \mathrm{Cr}, \mathrm{Pt} / \mathrm{Ti}$, and $\mathrm{Pt} / \mathrm{Ta}$. The fourth variant consists of a device without an adhesion layer; thus a layer of $150 \mathrm{~nm}$ is deposited directly on silicon nitride. This variant will be refererd to as $\mathrm{Pt} / \mathrm{SiN}$.

\footnotetext{
${ }^{a)}$ Electronic mail: zm@ecs.soton.ac.uk
}

All films were deposited in a dc magnetron sputtering system. The system has a rotating substrate holder, with a distance of about $18 \mathrm{~cm}$ between the substrate and target. After the deposition of the adhesion layers, the samples stayed in vacuo prior to the deposition of the platinum film. After patterning and etching, the resistors were released with dimensions of $1 \mathrm{~mm}$ in length and $4 \mu \mathrm{m}$ in width. Prior to experiments, the sensors were annealed at $500^{\circ} \mathrm{C}$. They consisted of two wires which are $200 \mu \mathrm{m}$ apart. The resistance of the wires was $300 \Omega$ at room temperature. As a reference in the experiments, a common metal film resistor of $300 \Omega$ was used. The output signal was amplified twice by two amplifiers with an amplification factor of $50 \mathrm{~dB}$. The signals were recorded by a 20 bits analog-to-digital converter with an input impedance of $30 \mathrm{k} \Omega$, and a flat frequency response in the bandwidth $(30 \mathrm{~Hz}, 20 \mathrm{kHz})$. The recorded signal was digitized and analyzed in a personal computer. The temperature of the wires was varied from 293 to $646 \mathrm{~K}$, and the spectrum of the detected output signal (in $\mathrm{V}^{2} / \mathrm{Hz}$ ) at each temperature was computed. In Fig. 1 we show the frequency dependence of the power spectrum of the voltage fluctuations for $\mathrm{Pt} / \mathrm{SiN}$ and $\mathrm{Pt} / \mathrm{Ta}$ at temperatures 400 and $477 \mathrm{~K}$, respectively. The power spectrum scales with frequency as $S_{V}(f) \sim 1 / f^{\alpha}$ for frequencies below $3 \mathrm{kHz}$ for $\mathrm{Pt} / \mathrm{Ta}$ and below $2 \mathrm{kHz}$ for $\mathrm{Pt} / \mathrm{SiN}$. The values of $\alpha$ are $\alpha \simeq 0.90$ and $\alpha \simeq 1.5$ for Pt/SiN and $\mathrm{Pt} / \mathrm{Ta}$, respectively. These values were determined by a regression fit on a double logarithmic plot of the power spectrum versus frequency. The flat portions of the spectrum correspond to white noise level. The value of $\alpha \simeq 1$ was also observed by Scofield et $a l^{2}$ for $142 \mathrm{~nm}$ thick platinum on sapphire at $400{ }^{\circ} \mathrm{C}$.

For all wires $(\mathrm{Pt} / \mathrm{Ta}, \mathrm{Pt} / \mathrm{Ti}, \mathrm{Pt} / \mathrm{Cr}$, and $\mathrm{Pt} / \mathrm{SiN})$ the power spectrum follows the power law scaling $S_{v}(f) \sim 1 / f^{\alpha}$. For wires with an adhesion layer, the scaling was observed over a range of two orders of magnitude in frequency and the value of the exponent $\alpha$ was found to be close to $3 / 2$. We found this exponent to be temperature independent (within the error range) over the considered temperature range, as shown in Fig. 2.

The values of $\alpha$ found in all wires with an adhesion layer are consistent with the value found for resistance fluctuations 


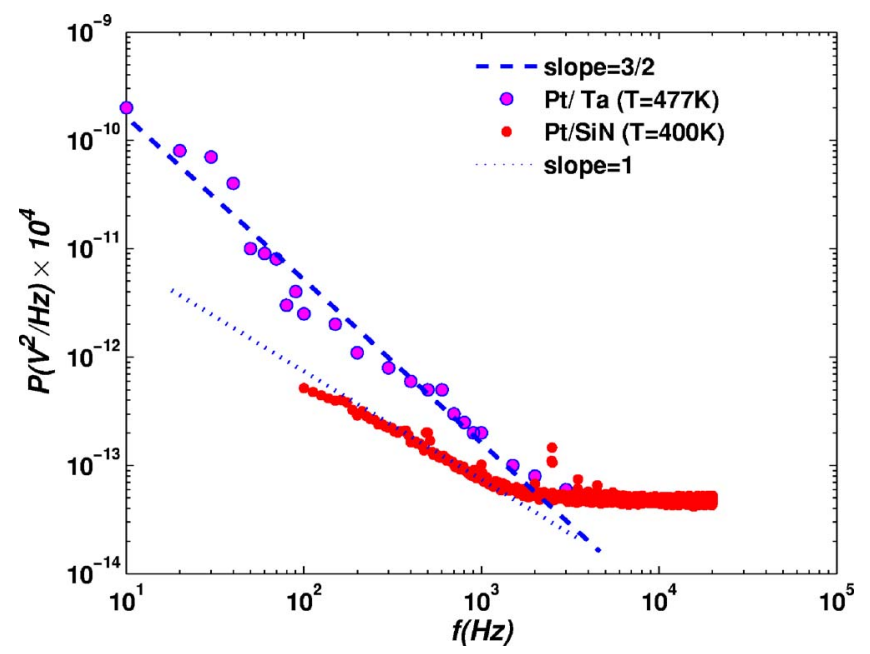

FIG. 1. (Color online) Voltage fluctuations power spectrum of Pt/Ta and $\mathrm{Pt} / \mathrm{SiN}$ systems at $T=477 \mathrm{~K}$ and $T=400 \mathrm{~K}$, respectively. The value of $\alpha$ $\simeq 3 / 2$ was common to all films with an adhesion layer. For comparison, we plot two lines with slopes $3 / 2$ and 1 , respectively. The linear regression fit to the data in the scaling range, gives slopes of $1.5 \pm 0.03$ and $0.9 \pm 0.02$ for $\mathrm{Pt} / \mathrm{Ta}$ and $\mathrm{Pt} / \mathrm{SiN}$, respectively.

in niobium films ${ }^{4} \alpha=3 / 2$. Scofield and $\mathrm{Webb}^{4}$ proposed that hydrogen diffusion in niobium films modulates the resistance of the films where fluctuations of the number of hydrogen ions in the film segment induce the fluctuations of the resistance, i.e., $\delta r \propto \delta N$, where the fluctuation $\delta N$ obeys the diffusion equation in the fixed volume of the film's segment. In the framework of this model, ${ }^{4}$ a diffusing species in the film of length $L$, will generate a power spectrum of the form $f S_{V}(f) /\left\langle\delta V^{2}\right\rangle=2 P(x)-P(2 x)$, where $x=\left(f / f_{c}\right)^{1 / 2}$, $f_{c}=D_{0} \exp \left(-E / K_{B} T\right) / \pi L^{2}$ and

$$
P(x)=\left(1-e^{-x}(\sin x+\cos x)\right) /(\pi x) .
$$

Here, $\left\langle\delta V^{2}\right\rangle$ is the voltage variance. We first assume that the fluctuations in the wires with the adhesion layer are described by the one dimensional diffusion process mentioned above and focus on the $\mathrm{Pt} / \mathrm{Ta}$ wires. To determine the characteristic frequency $f_{c}$ for $\mathrm{Pt} / \mathrm{Ta}$, we perform a nonlinear regression fit of the data to the theoretical expression of the one dimensional power spectrum (1) in the working frequency range of the sensor. In Fig. 3 we show the plot of

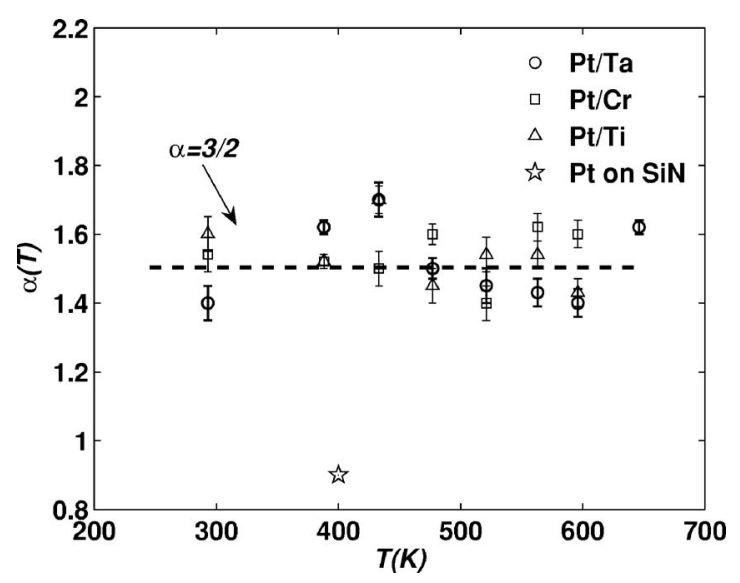

FIG. 2. Plot of the exponent $\alpha$ as a function of the temperature for wires with and without an adhesion layer. Wires having an adhesion layer have a value of $\alpha$ consistent with the value of $3 / 2$ arising from the long range diffusions. diffusions.
Downloaded 14 Sep 2007 to 152.78 .236 .138 . Redistribution subject to AlP liffusion model.

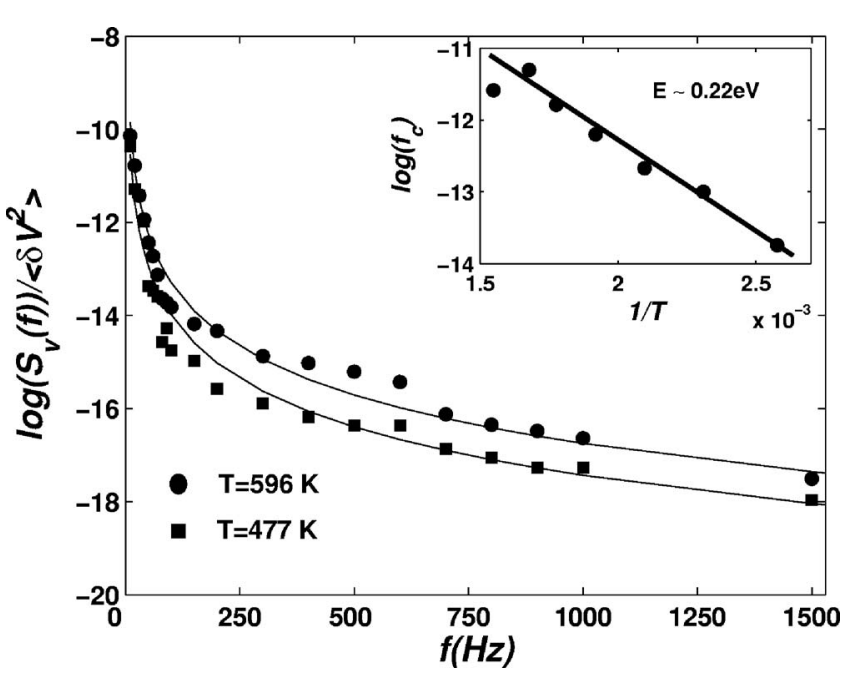

FIG. 3. Plot of $\log \left(S_{V}(f)\right) /\left\langle\delta V^{2}\right\rangle$ vs $\mathrm{f}$ for Pt/Ta. The solid lines are the fit to the theoretical power spectrum. The inset shows $\log \left(f_{c}\right)$ vs $1 / T$.

$\log \left(S_{V}(f)\right) /\left(\left\langle\delta V^{2}\right\rangle\right)$ versus $f$ as well as the theoretical fit to the data for two different temperatures $T=596 \mathrm{~K}$ and $T=477 \mathrm{~K}$. We have determined the characteristic frequency at various temperatures: $596,563,521,477$, and $433 \mathrm{~K}$. The inset of Fig. 3 shows the plot of the logarithm of $f_{c}$ versus $1 / T$, the slope gives the activation energy, and the intercept gives the diffusion coefficient preexponential factor for $\mathrm{Pt} / \mathrm{Ta}$, which are $E_{\mathrm{Pt} / \mathrm{Ta}} \simeq 0.22 \pm 0.04$ and $D_{0}$ $\simeq 2.4 \times 10^{-5} \mathrm{~cm}^{2} \mathrm{~s}^{-1}$ for $L=1 \mathrm{~mm}$. The value found for the activation energy is very close to the value derived directly from the semilogarithmic plot of $S_{V}(40 \mathrm{~Hz})$ (Fig. 5) as a function of $1 / T$, which is related to $E_{\mathrm{Pt} / \mathrm{Ta}}$, by the relation $E_{\mathrm{P} / \mathrm{Ta}}^{\prime}=(3 / 2) E_{\mathrm{Pt} / \mathrm{Ta}}$. We found $E_{\mathrm{P} / \mathrm{Ta}}^{\prime}=0.35 \pm 0.02 \mathrm{eV}$ which gives $E_{\mathrm{Pt} / \mathrm{Ta}}=0.23 \pm 0.01 \mathrm{eV}$. The evidence of the diffusion process can be shown by plotting $\log \left(f_{c} S_{V}(f, T)\right)$ versus $\log \left(f / f_{c}(T)\right)$ (Fig. 4) at different temperatures for $\mathrm{Pt} / \mathrm{Ta}$ wires, which shows a data collapse into a single curve, as expected from a diffusion process.

In Fig. 5 we plot the logarithm of the quantity $\Omega(T)=f^{\alpha} S_{V}(f)$ as a function of $1 / T$ at $40 \mathrm{~Hz}$. The plots corresponding to wires with an adhesion layer are reminiscent of a thermally activated process. The activation energies

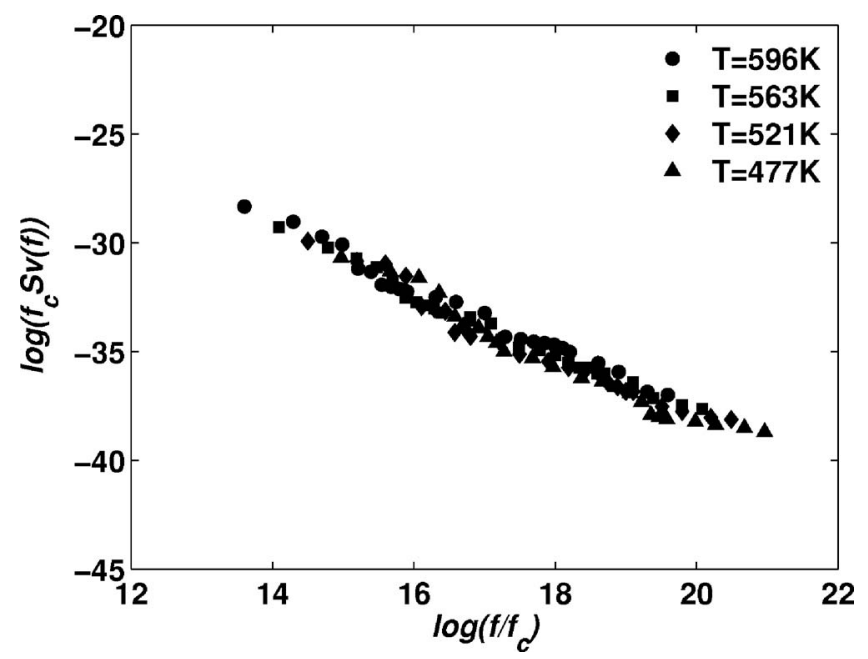

FIG. 4. Plots of $\log \left(f_{c} S_{V}(f, T)\right)$ vs $\log \left(f / f_{c}(T)\right)$ for Pt/Ta wire at different temperatures showing the collapse of the data on a single curve as predicted 


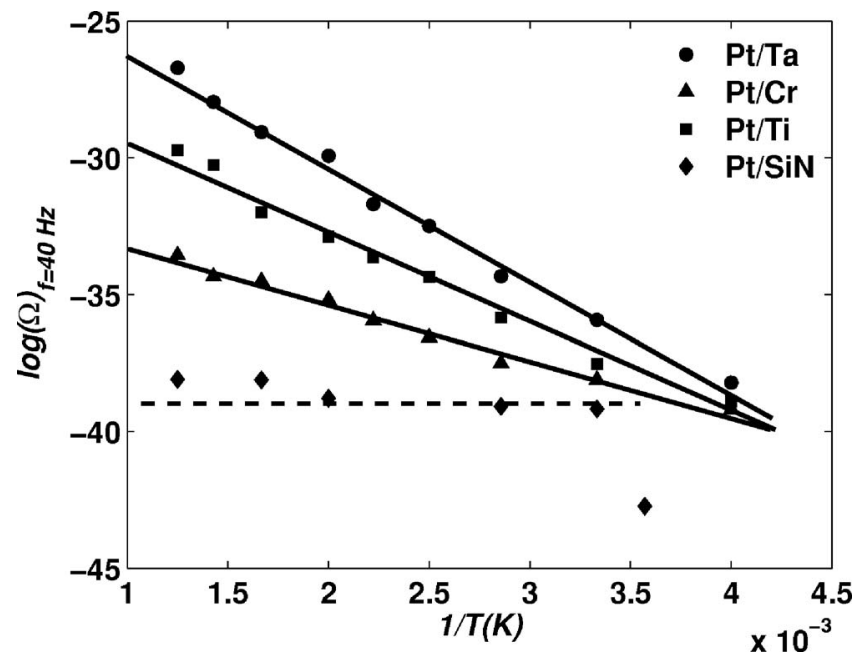

FIG. 5. Plots of $\Omega(T)=f^{\alpha} S_{V}(f)$ vs $1 / T$ showing a thermally activated process in films with an adhesion layer.

found for $\mathrm{Pt} / \mathrm{Ti}$ and $\mathrm{Pt} / \mathrm{Cr}$ wires are $0.190 \pm 0.02 \mathrm{eV}$ and $0.118 \pm 0.01 \mathrm{eV}$, respectively. No evidence of a thermally activated process was found in wires with no adhesion layer as clearly noticed in Fig. 5. The value of the activation energy found for the $\mathrm{Pt} / \mathrm{Ta}$ wires is very close to the value found in niobium films $(\simeq 0.23 \mathrm{eV})$, which was attributed to hydrogen diffusion in wires. ${ }^{4}$ The evidence of a diffusive process in wires with an adhesion layer suggests that impurities, mainly hydrogen and oxygen, modulate the wires resistance. In our case, a strong candidate for this process is oxygen since at high temperature (above $600 \mathrm{~K}$ ), hydrogen is easily effused off the wires and the exponent $\alpha$ still maintains its value of $3 / 2$. Ambient oxygen can freely penetrate the wires through the platinum layer through grain boundaries. All three wires with adhesion layers have in common the formation of oxides at the interface. In Pt/Ti films, ambient oxygen and titanium can diffuse through platinum films where they undergo chemical reactions to form $\mathrm{TiO}_{x}$ (Refs. 9 and 10) or $\mathrm{PtTi}_{x}$ components. It was also reported that for thin titanium layers, the titanium oxide phase is located at the boundaries of platinum grains. ${ }^{10}$ Tantalum hardly diffuses into the $\mathrm{Pt}$ layer and, consequently, the oxidation of Ta occurs mainly at the interface $\mathrm{Pt} / \mathrm{Ta}^{11}$ As a result, stress induced defects are less present in $\mathrm{Pt} / \mathrm{Ta}$ in comparison with $\mathrm{Pt} / \mathrm{Ti}$. Whereas chromium also has these effects (as for Pt/Ti), it also has a strong tendency to react with Pt to form an eutecticum, even at rather low temperatures. ${ }^{12}$ We believe that oxides formed at the interface act as scattering points and that different activation energies are associated with the different natures of oxide scatterers at the interface. The absence of the long range diffusion in the $\mathrm{Pt}$ without an adhesion layer suggests that there is no oxide formation at the interface and, consequently, no scattering process of oxygen. However, further investigations are needed to confirm this assumption. Nevertheless, the present work shows that there is less excess noise in $\mathrm{Pt}$ without an adhesion layer than in $\mathrm{Pt}$ with an adhesion layer. The values of the diffusion coefficient for $\mathrm{Pt} / \mathrm{Ta}$ provide evidence of an enhanced diffusion process, since these values are much larger than the values found for the bulk platinum. ${ }^{13}$

The authors would like to thank R. Tiggelaar for useful discussions.

${ }^{1}$ P. Dutta and P. M. Horn, Rev. Mod. Phys. 53, 497 (1981).

${ }^{2}$ J. H. Scofield, J. V. Mantese, and W. W. Webb, Phys. Rev. B 32, 736 (1985).

${ }^{3}$ M. B Weissman, Rev. Mod. Phys. 60, 537 (1988).

${ }^{4}$ J. H. Scofield and W. W. Webb, Phys. Rev. Lett. 54, 353 (1985).

${ }^{5}$ B. D. Nevins and M. B. Weissman, Phys. Rev. B 41, 1301 (1990).

${ }^{6}$ J. H. Scofield and W. W. Webb, Noise in Physical Systems and $1 / \mathrm{f}$ Noise, edited by M. Savelli, G. Lecoy, and J. P. Nougier (North-Holland, Amsterdam, 1983), p. 405.

${ }^{7}$ S. Kar and A. K. Raychaudhuri, Appl. Phys. Lett. 81, 5165 (2002).

${ }^{8}$ H. de Bree, P. Leussink, T. Korthorst, H. Jansen, T. S. J. Lammerink, and M. Elwenspoek, Sens. Actuators, A 54, 552 (1996).

${ }^{9}$ J. O. Olowolafe, R. E. Jones, Jr., A. C. Campbell, R. I. Hegde, C. J. Mogab, and R. B. Gregory, J. Appl. Phys. 73, 1764 (1993).

${ }^{10}$ S.-K. Ko, N. M. Devashrajee, and S. P. Murarka, Mater. Res. Soc. Symp. Proc. 260, 665 (1992).

${ }^{11}$ T. Maeder, L. Sagalowicz, and P. Muralt, Jpn. J. Appl. Phys. 1, 2007 (1998).

${ }^{12}$ C. J. Terblanche, J. P. Roux, P. E. Viljoen, and H. C. Swart, Appl. Surf. Sci. 78, 275 (1994).

${ }^{13}$ R. Schmiedl, V. Demuth, P. Lahnor, H. Godehardt, Y. Bodschwinna, C. Harder, L. Hammer, H.-P. Strunk, M. Schulz, and K. Heinz, Appl. Phys. A: Mater. Sci. Process. 62, 223 (1996). 